\title{
Nonperturbative algorithm for the resistive wall impedance of general cross-section beam pipes
}

\author{
Alexandru Macridin, Panagiotis Spentzouris, and James Amundson \\ Fermilab, P.O. Box 500, Batavia, Illinois 60510, USA \\ (Received 19 September 2013; published 5 December 2013)
}

\begin{abstract}
We present an algorithm for calculating the impedance of infinitely long beam pipes with arbitrary cross section. The method is not restricted to ultrarelativistic beams or perturbative approximations with respect to the wall surface impedance or skin penetration depth. We exemplify our algorithm with a calculation of the impedance for rectangular metallic beam pipes. Unlike the situation in the perturbative regime, where the beam pipe geometry modifies the metallic resistive wall impedances by only a multiplicative factor, the beam pipe geometry has a more complex influence on the impedance when nonultrarelativistic effects are significant and in the ultrarelativistic regime at both small and large frequencies. Since our algorithm requires the boundary conditions at the beam pipe wall to be provided as linear relations between the transverse components of the electromagnetic field, we discuss a general algorithm to calculate these boundary conditions for multilayer beam pipes with arbitrary cross section.
\end{abstract}

DOI: 10.1103/PhysRevSTAB.16.121001

PACS numbers: 29.27.Bd, 29.27. - a, 41.75.- $-\mathrm{i}$

\section{INTRODUCTION}

Impedance plays an important role in the beam dynamics of high intensity accelerators, being a leading cause for losses and instabilities. There is vast literature addressing impedance calculations in accelerators. See, for example, [1-3] and the references therein. With a few exceptions, the vast majority of impedance studies address cylindrical [3-12] and parallel-plane [13-16] beam pipes. Since these systems are highly symmetric, characteristic modes can be decoupled and analytical expressions for the impedance can be derived. Of particular interest is the calculation of impedance in multilayer beam pipes; the problem has been addressed in the literature for both cylindrical [8-12] and parallel-plane $[14,15]$ geometries. Beam pipes of general cross section have also been addressed in the literature [17-19], but only in the ultrarelativistic approximation and for single-layered metallic pipes in the frequency region where perturbation theory with respect to the penetration skin depth is valid.

In this paper, we present a method for calculating the resistive wall impedance for infinitely long beam pipes with general cross section. Unlike previous investigations, our method works for systems with large wall surface impedances and in the nonperturbative regime for metallic pipes at both small and large frequencies. Another important difference from the existing literature is that our method does not impose an ultrarelativistic approximation. The ability to calculate the impedance for nonultrarelativistic beams and for systems with large wall surface impedance is extremely important for machines like the Fermilab Booster synchrotron, which has laminated magnets characterized by very

Published by the American Physical Society under the terms of the Creative Commons Attribution 3.0 License. Further distribution of this work must maintain attribution to the author(s) and the published article's title, journal citation, and DOI. large surface impedance $[13,16]$ and an injection energy of $400 \mathrm{MeV}(\gamma=1.42)$.

To illustrate our algorithm, we calculate the impedance of a rectangular metallic beam pipe, for both ultrarelativistic and finite- $\gamma$ cases. The ultrarelativistic perturbative regime is in perfect agreement with the work of Yokoya [18], which showed that the rectangular beam pipe impedance has a behavior similar to that of the circular and the parallel-plane geometries, the difference being only a renormalizing factor. However, we find that this simple renormalization is not valid at small and large frequencies, nor is it valid in the frequency regions where the nonultrarelativistic effects are noticeable.

The algorithm assumes that the electromagnetic field boundary conditions at the pipe walls are known and are provided as linear relations between the field transverse components. An example is the boundary conditions provided via the wall surface impedances. We discuss how the boundary conditions can be calculated for multilayer beam pipes of arbitrary cross section, using a similar numerical method to that used for calculating the impedance.

In order to check the correctness of our code, we compare the simulations with the analytical results for the parallelplane pipe impedance. Since, to our knowledge, the expressions for the nonultrarelativistic parallel-plane impedance as a function of wall surface impedances were never published, we present briefly their calculation in here.

The paper is organized as follows. The impedance algorithm is derived in Sec. II. In Sec. III the impedance of the rectangular beam pipe is calculated. Conclusions are presented in Sec. IV. In Appendix A an algorithm designed to calculate the electromagnetic field boundary conditions in multilayer beam pipes of arbitrary cross section is discussed. In Appendix B we present a derivation of the nonultrarelativistic impedance for the parallel-plane beam pipe. Appendix $\mathrm{C}$ presents a modified version of 
the impedance calculation algorithm which might be useful for numerical optimization.

\section{FORMALISM}

Inside the vacuum beam pipe, the electric and magnetic fields are given by

$$
\vec{E}=-\nabla \Phi-\frac{\partial \vec{A}}{\partial t}
$$

and

$$
Z_{0} \vec{H}=c \nabla \times \vec{A},
$$

where $\Phi$ and $\vec{A}$ are the electric and magnetic vector potentials, respectively. The equations for the potentials in the vacuum beam pipe are

$$
\begin{gathered}
\nabla^{2} \Phi-\frac{1}{c^{2}} \frac{\partial^{2} \Phi}{\partial t^{2}}=-\frac{\rho}{\epsilon_{0}}, \\
\nabla^{2} \vec{A}-\frac{1}{c^{2}} \frac{\partial^{2} \vec{A}}{\partial t^{2}}=-\mu_{0} \vec{j}, \\
\nabla \vec{A}+\frac{1}{c^{2}} \frac{\partial \Phi}{\partial t}=0 .
\end{gathered}
$$

Equation (5) is the Lorentz gauge condition. Within the Lorentz gauge constraint, the potentials can undergo a gauge transformation,

$$
\begin{aligned}
\vec{A}^{\prime} & =\vec{A}-\nabla \chi, \\
\Phi^{\prime} & =\Phi+\frac{\partial \chi}{\partial t},
\end{aligned}
$$

with the gauge field satisfying

$$
\nabla^{2} \chi-\frac{1}{c^{2}} \frac{\partial^{2} \chi}{\partial t^{2}}=0
$$

The impedance describes the response of a witness particle to the electromagnetic field created in the accelerator walls by a source particle. We assume a source particle with a transverse offset $\left(x_{0}, y_{0}\right)$ moving in the $z$ (longitudinal) direction with velocity $\beta c$. The charge density and electric current are given by

$$
\begin{aligned}
\rho(x, y, z, t) & =\rho(x, y, z-\beta c t) \\
& =\rho \delta\left(x-x_{0}\right) \delta\left(y-y_{0}\right) e^{i(\omega t-k z),} \\
\vec{j}(x, y, z, t)= & \vec{j}(x, y, z-\beta c t) \\
= & \rho \delta\left(x-x_{0}\right) \delta\left(y-y_{0}\right) \beta c \hat{z} e^{i(\omega t-k z)} .
\end{aligned}
$$

We are looking for synchronous solutions

$$
\begin{aligned}
& \vec{E}(x, y, z, t)=\vec{E}(x, y) e^{i(\omega t-k z)}, \\
& \vec{H}(x, y, z, t)=\vec{H}(x, y) e^{i(\omega t-k z)},
\end{aligned}
$$

where $k=\frac{\omega}{\beta c}$.
The impedance terms are defined as a derivative of a given order of the electromagnetic force acting on the witness particle with respect to the source or/and witness particle displacement. Here we consider only the zerothand first-order terms.

We assume the following definitions: The zeroth-order longitudinal impedance is

$$
Z^{\|}=-\frac{F_{z}}{q \rho \beta c}\left(x=y=x_{0}=y_{0}=0\right) .
$$

The first-order horizontal transverse impedances are

$$
\begin{aligned}
& Z_{x}^{w}=-\frac{1}{i q \rho \beta c} \frac{\partial F_{x}}{\partial x}\left(x=y=x_{0}=y_{0}=0\right), \\
& Z_{x}^{s}=-\frac{1}{i q \rho \beta c} \frac{\partial F_{x}}{\partial x_{0}}\left(x=y=x_{0}=y_{0}=0\right),
\end{aligned}
$$

where $Z_{x}^{w}\left(Z_{x}^{s}\right)$ describes the effect proportional to the displacement of the witness (source) particle. It is customary to define the transverse impedance with a factor of $i$ [4].

For beam pipes with low symmetry it is possible that a vertically displaced source particle kicks the witness particle in the horizontal plane or that a vertical displaced witness particle is kicked horizontally by a term proportional to its vertical displacement [20]. Correspondingly, the following transverse impedances can be defined:

$$
\begin{aligned}
& Z_{x}^{s y}=-\frac{1}{i q \rho \beta c} \frac{\partial F_{x}}{\partial y_{0}}\left(x=y=x_{0}=y_{0}=0\right), \\
& Z_{x}^{w y}=-\frac{1}{i q \rho \beta c} \frac{\partial F_{x}}{\partial y}\left(x=y=x_{0}=y_{0}=0\right) .
\end{aligned}
$$

Similar equations can be written for the vertical impedances.

\section{A. Potential field equations}

In Fourier space $(x, y, k, \omega)$, for the charge and the current given by Eqs. (9) and (10), the potential equations, Eqs. (3) and (4), read

$$
\begin{gathered}
\frac{\partial^{2} \Phi}{\partial x^{2}}+\frac{\partial^{2} \Phi}{\partial y^{2}}-k_{r}^{2} \Phi=-\frac{\rho}{\epsilon_{0}} \delta\left(x-x_{0}\right) \delta\left(y-y_{0}\right), \\
\frac{\partial^{2} \vec{A}}{\partial x^{2}}+\frac{\partial^{2} \vec{A}}{\partial y^{2}}-k_{r}^{2} \vec{A}=-\frac{\beta}{c} \frac{\rho}{\epsilon_{0}} \delta\left(x-x_{0}\right) \delta\left(y-y_{0}\right) \hat{z},
\end{gathered}
$$

where

$$
k_{r}^{2}=k^{2}-\frac{\omega^{2}}{c^{2}}=k^{2}\left(1-\beta^{2}\right)=\frac{k^{2}}{\gamma^{2}} .
$$

It is convenient to eliminate the calculation of the $z$ component of the vector potential by fixing the gauge such that 


$$
A_{z}=\frac{\beta}{c} \Phi
$$

This, together with the Lorentz gauge constraint, Eq. (5), yields the following equation for the remaining components of the vector potential:

$$
\partial_{x} A_{x}+\partial_{y} A_{y}=0 .
$$

By employing Green's Theorem [21], the solution for Eqs. (18) and (19) can be written formally as

$$
\begin{gathered}
\Phi(x, y)=\Phi_{0}(x, y)+\oint D\left(x, y ; r_{l}\right) \Phi\left(r_{l}\right) d l \\
-\oint G\left(x, y ; r_{l}\right) \partial_{n} \Phi\left(r_{l}\right) d l, \\
A_{x, y}(x, y)=\oint D\left(x, y ; r_{l}\right) A_{x, y}\left(r_{l}\right) d l-\oint G\left(x, y ; r_{l}\right) \partial_{n} A_{x, y}\left(r_{l}\right) d l,
\end{gathered}
$$

where the one-dimensional integrals are taken along the beam pipe contour in the transverse plane and

$$
\begin{gathered}
G\left(x, y ; x^{\prime}, y^{\prime}\right)=-\frac{1}{2 \pi} K_{0}\left(k_{r} R\right), \\
R=\sqrt{\left(x-x^{\prime}\right)^{2}+\left(y-y^{\prime}\right)^{2}}
\end{gathered}
$$

is the Green function satisfying

$$
\frac{\partial^{2} G}{\partial x^{2}}+\frac{\partial^{2} G}{\partial y^{2}}-k_{r}^{2} G=\delta\left(x-x^{\prime}\right) \delta\left(y-y^{\prime}\right) .
$$

$K_{0}$ is the modified Bessel function of the second kind, $\Phi_{0}$ is the free space (i.e. no beam pipe) solution

$$
\begin{aligned}
\Phi_{0}(x, y) & =-\int G\left(x, y ; x^{\prime}, y^{\prime}\right) \frac{\rho\left(x^{\prime}, y^{\prime}\right)}{\epsilon_{0}} d x^{\prime} d y^{\prime} \\
& =\frac{\rho}{2 \pi \epsilon_{0}} K_{0}\left(k_{r} \sqrt{\left(x-x_{0}\right)^{2}+\left(y-y_{0}\right)^{2}}\right),
\end{aligned}
$$

and

$$
D\left(x, y ; r_{l}\right)=\partial_{n} G\left(x, y ; r_{l}\right),
$$

where the normal derivative $\partial_{n}$ is taken in $r_{l}$, i.e., on the wall contour, and outward.

\section{Discretized equations}

Equations (23) and (24) show that the solution is determined once the potentials and their normal components on the beam pipe's wall are known. Our algorithm finds the solution numerically, by taking $N$ points at position $r_{i}$ on the contour. The discretized equations for the surface potentials $\Phi\left(r_{i}\right) \equiv \bar{\Phi}_{i}$ and $\partial_{n} \Phi\left(r_{i}\right) \equiv \overline{\partial \Phi}_{i}$ are

$$
\bar{\Phi}_{i}=\bar{\Phi}_{0 i}+\sum_{j=0}^{N-1}\left(D_{i, j} \bar{\Phi}_{j}-G_{i, j} \overline{\partial \Phi}_{j}\right)
$$

$$
\begin{aligned}
& \bar{A}_{x i}=\sum_{j=0}^{N-1}\left(D_{i, j} \bar{A}_{x j}-G_{i, j} \overline{\partial A}_{x j}\right), \\
& \bar{A}_{y i}=\sum_{j=0}^{N-1}\left(D_{i, j} \bar{A}_{y j}-G_{i, j} \overline{\partial A}_{y j}\right) .
\end{aligned}
$$

The bars over the potentials indicate in our notation that they are evaluated on the surface contour. The equations can be written in a compact matrix form:

$$
\begin{gathered}
\bar{\Phi}=\bar{\Phi}_{0}+D \bar{\Phi}-G \overline{\partial \Phi}, \\
\bar{A}_{x}=D \bar{A}_{x}-G \bar{\partial}_{x}, \\
\bar{A}_{y}=D \bar{A}_{y}-G \overline{\partial A}_{y} .
\end{gathered}
$$

We have $6 N$ variables, $\bar{\Phi}_{i}, \bar{A}_{x i}, \bar{A}_{y i}, \overline{\partial \Phi}_{i}, \overline{\partial A}_{x i}, \overline{\partial A}_{y i}$, $i=\overline{1, N}$, and $3 N$ equations, Eqs. (30)-(32). The gauge fixing condition, Eq. (22), and the field boundary conditions provide the other set of $3 N$ equations required to solve the problem. A straightforward way to solve the problem is to consider all $6 \mathrm{~N}$ independent variables and to reduce the problem to a system of $6 \mathrm{~N}$ complex linear equations, as described in Appendix C. However, it is possible reduce the problem to a set of $2 \mathrm{~N}$ linear equations. From Eqs. (30)-(32) one can write the potentials' normal derivatives as function of the potentials,

$$
\begin{gathered}
\overline{\partial \Phi}=U \bar{\Phi}+\overline{\partial \Phi}^{\infty}, \\
\overline{\partial A}_{x}=U \bar{A}_{x}, \\
\overline{\partial A}_{y}=U \bar{A}_{y},
\end{gathered}
$$

where

$$
U=G^{-1}(D-I)
$$

and

$$
\overline{\partial \Phi}^{\infty}=G^{-1} \bar{\Phi}_{0} .
$$

$\overline{\partial \Phi}^{\infty}$ is the normal derivative of the potential of a perfectly conducting beam pipe (with conductivity $\sigma=\infty$ ).

So far the $3 N$ surface potentials $\left(\bar{\Phi}_{i}, \bar{A}_{x i}, \bar{A}_{y i}\right), i=\overline{1, N}$, have been considered as independent variables. The gauge fixing constraint, Eq. (22), eliminates one more set of $N$ variables. Equation (22) can be written as a function of the normal and tangential derivative of the vector potentials. By considering the discretized tangential derivative of the potential to the surface to be

$$
\partial_{\|} \bar{\Phi}_{i}=\frac{\bar{\Phi}_{i+1}-\bar{\Phi}_{i-1}}{2 h_{i}},
$$

where $2 h_{i}$ is the distance on the surface between the points $r_{i+1}$ and $r_{i-1}$, one can write the tangential derivative matrix as 


$$
\partial_{\|}(i, j)=\frac{1}{2 h_{i}}\left(\delta_{i, j+1}-\delta_{i, j-1}\right) .
$$

However, depending on the surface characteristics, a more suitable discretization of the tangential derivative can be chosen. At any point $r_{i}$, the surface is characterized by a tangential vector $\vec{t}_{i}=t_{x i} \vec{i}+t_{y i} \vec{j}$ and a surface normal vector $\vec{n}_{i}=n_{x i} \vec{i}+n_{y i} \vec{j}$. Equation (22) on the surface reads

$$
\left(t_{x} \partial_{\|}+n_{x} \partial_{n}\right) \bar{A}_{x}+\left(t_{y} \partial_{\|}+n_{y} \partial_{n}\right) \bar{A}_{y}=0 .
$$

Employing Eqs. (37) and (38) the gauge constraint becomes

$$
\left(t_{x} \partial_{\|}+n_{x} U\right) \bar{A}_{x}+\left(t_{y} \partial_{\|}+n_{y} U\right) \bar{A}_{y}=0 .
$$

Equation (44) allows us to write $\bar{A}_{x}$ and $\bar{A}_{y}$ as a function of single independent variable $\bar{A}$, thus

$$
\begin{aligned}
& \bar{A}_{x}=L_{x} \bar{A}, \\
& \bar{A}_{y}=L_{y} \bar{A} .
\end{aligned}
$$

For example, one can choose $\bar{A}_{x}$ as the independent variable and express $\bar{A}_{y}$ as a function of $\bar{A}_{x}$ :

$$
\begin{gathered}
\bar{A}=\bar{A}_{x}, \\
L_{x}=I, \\
L_{y}=-\left(t_{y} \partial_{\|}+n_{y} U\right)^{-1}\left(t_{x} \partial_{\|}+n_{x} U\right) .
\end{gathered}
$$

However, other choices might be more convenient, depending on the particular problem.

We reduced the number of independent variables to $2 N$, $\left(\bar{\Phi}_{i}, \bar{A}_{i}\right), i=\overline{1, N}$. They are to be determined from the continuity conditions of the tangential fields at the wall. Using the potential equations, Eqs. (36)-(38), (45), and (46) in Eqs. (1) and (2), the fields at the wall become

$$
\begin{gathered}
\bar{E}_{z}=i \frac{k}{\gamma^{2}} \bar{\Phi} \\
\bar{E}_{t}=-\partial_{\|} \bar{\Phi}-i \omega\left(t_{x} L_{x}+t_{y} L_{y}\right) \bar{A} \\
Z_{0} \bar{H}_{z}=c\left[\left(t_{x} \partial_{\|}+n_{x} U\right) L_{y}-\left(t_{y} \partial_{\|}+n_{y} U\right) L_{x}\right] \bar{A} \\
Z_{0} \bar{H}_{t}=\beta(\vec{t} \times \vec{n})_{z} U \bar{\Phi}+i k c\left(t_{x} L_{y}-t_{y} L_{x}\right) \bar{A}+\beta(\vec{t} \times \vec{n})_{z} \overline{\partial \Phi^{\infty}} .
\end{gathered}
$$

Our algorithm assumes the boundary conditions form of a system of $2 N$ linear equations,

$$
\begin{aligned}
& \bar{E}_{z}=\mathcal{R}_{11} \bar{H}_{z}+\mathcal{R}_{12} \bar{H}_{t}, \\
& \bar{E}_{t}=\mathcal{R}_{21} \bar{H}_{z}+\mathcal{R}_{22} \bar{H}_{t},
\end{aligned}
$$

where the matrix $\mathcal{R}$ elements depend on the wall geometry and on the electromagnetic properties of the medium outside the beam pipe. Often the boundary conditions can be determined as an independent problem. We present an algorithm for determining the boundary conditions for a multilayer beam pipe with arbitrary cross section in Appendix A.

As an example, assume that the wall surface impedances,

$$
\begin{aligned}
& \mathcal{R}_{z}=\frac{\bar{E}_{z}}{\bar{H}_{t}}, \\
& \mathcal{R}_{t}=\frac{\bar{E}_{t}}{\bar{H}_{z}},
\end{aligned}
$$

are known at every point on the surface. This would correspond to $\mathcal{R}_{11 i j}=\delta_{i j} \mathcal{R}_{z}, \mathcal{R}_{12}=\mathcal{R}_{21}=0$, and $\mathcal{R}_{22 i j}=$ $\delta_{i j} \mathcal{R}_{t}$. These boundary conditions are specific to metallic beam pipes characterized by large conductivity. The equations for $(\bar{\Phi}, \bar{A})$ become

$$
\begin{gathered}
\partial_{\|} \bar{\Phi}+\left[i \omega\left(t_{x} L_{x}+t_{y} L_{y}\right)+\frac{\mathcal{R}_{t}}{Z_{0}} c\left(t_{x} \partial_{\|}+n_{x} U\right) L_{y}\right. \\
\left.-\left(t_{y} \partial_{\|}+n_{y} U\right) L_{x}\right] \bar{A}=0, \\
\left(i \frac{k}{\gamma^{2}}-\frac{\mathcal{R}_{z}}{Z_{0}} \beta(\vec{t} \times \vec{n})_{z} U\right) \bar{\Phi}-i k c \frac{\mathcal{R}_{z}}{Z_{0}}\left(t_{x} L_{y}-t_{y} L_{x}\right) \bar{A} \\
=\frac{\mathcal{R}_{z}}{Z_{0}} \beta(\vec{t} \times \vec{n})_{z} \overline{\partial \Phi^{\infty}} .
\end{gathered}
$$

The problem reduces to the linear equation

$$
M P=S,
$$

where $M$ is a complex $2 N \times 2 N$ matrix and $P=(\bar{\Phi}, \bar{A})$ and $S \propto \overline{\partial \Phi}^{\infty} \propto \bar{\Phi}_{0}$ are vectors of size $2 N$. For our choice of the boundary conditions given by Eqs. (56) and (57), $S=\left(0, \frac{\mathcal{R}_{z}}{Z_{0}} \beta G^{-1} \bar{\Phi}_{0}\right)$.

For beam pipes with specific symmetries, the number of independent variables can be reduced by a factor equal to the number of symmetries. For example, for the calculation of the longitudinal impedance in a rectangular beam pipe, the size of the problem can be reduced by a factor of 4 . For the calculation of the rectangular transverse impedances, which require an off-centered source along one transverse direction, the size of the problem can be reduced by a factor of 2 .

Many applications, such as numerical beam dynamics simulations, require knowledge of the contribution of the wall finite conductivity to the impedance. For this it is necessary to subtract the contribution corresponding to the perfectly conducting wall. For an ideal conductor

$$
\Phi^{\infty}(x, y)=\Phi_{0}(x, y)-\sum_{j} G\left(x, y ; r_{j}\right) \bar{\partial}_{j}^{\infty} .
$$

The wall finite-conductivity contribution to the potential is 


$$
\begin{aligned}
\Phi^{\sigma}(x, y) & =\Phi(x, y)-\Phi^{\infty}(x, y) \\
& =\sum_{j}\left[D\left(x, y ; r_{j}\right) \bar{\Phi}_{j}-G\left(x, y ; r_{j}\right)\left(\overline{\partial \Phi}_{j}-\overline{\partial \Phi}_{j}^{\infty}\right)\right] .
\end{aligned}
$$

We would like to highlight a subtlety in the calculation of the discrete Green function matrices $G$ and $D$. These matrices connect points along the wall surface and should be derived from Eq. (23) by taking proper limit when the wall is approached from inside. Since $G(R)$ is singular for $R=0$, and at small $R$

$$
G(R) \propto-K_{0}\left(k_{r} R\right) \approx \ln \frac{k_{r} R}{2}+\gamma_{e},
$$

where $\gamma_{e}=0.57721$ is the Euler's constant, we take

$G_{i i}=-\frac{1}{2 \pi} 2 \int_{0}^{\frac{h_{i}}{2}} d s K_{0}\left(k_{r} s\right)=\frac{h_{i}}{2 \pi}\left(\ln \frac{k_{r} h_{i}}{4}-1-\gamma_{e}\right)$.

A careful examination of

$$
\lim _{\epsilon \rightarrow 0} \int D\left(\vec{r}-\vec{n} \epsilon, \vec{r}^{\prime}\right) \Phi\left(\vec{r}^{\prime}\right) d \vec{r}^{\prime}=\frac{1}{2} \Phi(\vec{r})
$$

when $\vec{r}$ is on the integration contour shows that

$$
D_{i i}=\frac{1}{2} \text {. }
$$

\section{B. Impedances}

The forces acting on the witness particle are

$$
\begin{gathered}
F_{z}=q E_{z}=q i \frac{k}{\gamma^{2}} \Phi, \\
F_{x}=q\left(E_{x}-\beta Z_{0} H_{y}\right)=-\frac{q}{\gamma^{2}} \partial_{x} \Phi, \\
F_{y}=q\left(E_{y}+\beta Z_{0} H_{x}\right)=-\frac{q}{\gamma^{2}} \partial_{y} \Phi .
\end{gathered}
$$

The impedances defined in Eqs. (13)-(17) become

$$
\begin{gathered}
Z^{\|}=-i Z_{0} \frac{k}{\gamma^{2} \beta} \frac{\epsilon_{0}}{\rho} \Phi\left(x=y=x_{0}=y_{0}=0\right), \\
Z_{x}^{w}=-Z_{0} \frac{i}{\gamma^{2} \beta} \frac{\epsilon_{0}}{\rho} \frac{\partial^{2} \Phi}{\partial x^{2}}\left(x=y=x_{0}=y_{0}=0\right), \\
Z_{x}^{s}=-Z_{0} \frac{i}{\gamma^{2} \beta} \frac{\epsilon_{0}}{\rho} \frac{\partial^{2} \Phi}{\partial x \partial x_{0}}\left(x=y=x_{0}=y_{0}=0\right), \\
Z_{x}^{s y}=-Z_{0} \frac{i}{\gamma^{2} \beta} \frac{\epsilon_{0}}{\rho} \frac{\partial^{2} \Phi}{\partial x \partial y_{0}}\left(x=y=x_{0}=y_{0}=0\right),
\end{gathered}
$$

$$
Z_{x}^{w y}=-Z_{0} \frac{i}{\gamma^{2} \beta} \frac{\epsilon_{0}}{\rho} \frac{\partial^{2} \Phi}{\partial x \partial y}\left(x=y=x_{0}=y_{0}=0\right) .
$$

The calculation of $Z_{x}^{w}$ and $Z_{x}^{w y}$ requires the derivatives of the potential at the witness particle position

$$
\frac{\partial^{2} \Phi}{\partial x^{2}}=\sum_{j}\left(\frac{\partial^{2} D\left(x, y ; r_{j}\right)}{\partial x^{2}} \bar{\Phi}_{j}-\frac{\partial^{2} G\left(x, y ; r_{j}\right)}{\partial x^{2}} \bar{\partial}_{j}\right)
$$

and

$$
\frac{\partial^{2} \Phi}{\partial x \partial y}=\sum_{j}\left(\frac{\partial^{2} D\left(x, y ; r_{j}\right)}{\partial x \partial y} \bar{\Phi}_{j}-\frac{\partial^{2} G\left(x, y ; r_{j}\right)}{\partial x \partial y} \overline{\partial \Phi}_{j}\right) .
$$

Note that only the solution of Eq. (60) for a centered source is required, as is the case for the longitudinal impedance. Thus, the calculation of the transverse impedance due to the witness particle displacement requires very small extra computational effort after the longitudinal impedance has been calculated and $(\bar{\Phi}, \overline{\partial \Phi})$ determined.

Calculation of $Z_{x}^{s}$ requires the derivation with respect to the source particle position

$$
\frac{\partial^{2} \Phi}{\partial x \partial x_{0}}=\sum_{j}\left(\frac{\partial D\left(x, y ; r_{j}\right)}{\partial x} \frac{\partial \bar{\Phi}_{j}}{\partial x_{0}}-\frac{\partial G\left(x, y ; r_{j}\right)}{\partial x} \frac{\partial\left(\overline{\partial \Phi}_{j}\right)}{\partial x_{0}}\right) .
$$

Since the potentials on the contour are found by solving a linear equation, the derivative with respect to $x_{0}$ can be found by solving

$$
M \frac{\partial P}{\partial x_{0}}=\frac{\partial S}{\partial x_{0}},
$$

where $\frac{\partial P}{\partial x_{0}}=\left(\frac{\partial \bar{\Phi}}{\partial x_{0}}, \frac{\partial \bar{A}}{\partial x_{0}}\right)$. Similar equations can be written for $\frac{\partial P}{\partial y_{0}}$ which is required for calculating $Z_{x}^{s y}$. The calculation of the transverse impedances caused by the source particle displacement requires solving $2 \mathrm{~N}$ linear complex equations which are different from the one corresponding to the longitudinal impedance.

\section{RECTANGULAR PIPE IMPEDANCE}

\section{A. Discussion}

The metallic beam pipes are characterized by large conductivity and, implicitly, by small surface impedance

$$
\mathcal{R}_{z}=\frac{1+i}{\delta \sigma}=(1+i) \sqrt{\frac{\omega \mu}{2 \sigma}},
$$

where $\delta$ is the penetration skin depth. Therefore the firstorder approximation in $\mathcal{R}_{z}$ works very well in the ultrarelativistic limit in the frequency region relevant for most beam dynamics problems. In this approximation the impedance is proportional to $\mathcal{R}_{z}$. The approximation fails at small frequencies when 


$$
\frac{\mathcal{R}_{z}}{Z_{0} k b} \gtrsim 1 \quad \text { or } \quad k \lesssim \frac{\mu_{r}}{\mu_{0} c \sigma b^{2}},
$$

and at large frequency when

$$
\frac{\mathcal{R}_{z}}{Z_{0}} k b \gtrsim 1 \quad \text { or } \quad k \gtrsim\left(\frac{\mu_{0} c \sigma}{b^{2} \mu_{r}}\right)^{\frac{1}{3}},
$$

as can be deduced from the analytical expression of the impedance for the circular and parallel-plane geometries $[13,16]$. For typical metallic pipes the small-frequency regime is relevant for distances larger than $z \gtrsim$ $10^{6}-10^{7} \mathrm{~m}$, while the large frequency regime is relevant at short range, $z \lesssim 10-100 \mu \mathrm{m}$. One might argue that these length scales make only the perturbative region of interest for beam dynamics. However, note that the short (long) length scale is proportionally increased (decreased) by the increase in the wall surface impedance, as can be inferred from Eqs. (80) and (81). The wall surface impedance can easily be increased by orders of magnitude by increasing the magnetic permeability and/or by reducing the conductivity. More complicated structures, like laminated chambers, are also characterized by orders of magnitude higher wall surface impedance $[13,16]$.

The small-frequency regime is relevant for large distance effects such as coherent tune shift in chambers with low symmetries [22]. The methods for impedance calculations for beam pipes with arbitrary cross section described in [17] and in [18] do not address this region.

The large frequency regime is relevant for short bunches; it is called the short-range resistive wall regime in the literature [23]. For circular chambers in the ultrarelativistic limit, Bane [23] showed that the impedance in the large frequency regime can be modeled by a low- $Q$ resonator. The method described in [17] does not address this region. Yokoya's algorithm [18] addresses the large frequency regime but neglects the contribution of the tangential surface impedance $\mathcal{R}_{t}$. While the contribution of $\mathcal{R}_{t}$ to the coupling impedance is small in the ultrarelativistic limit, it becomes important in the nonultrarelativistic regime at large frequencies. By inspecting the analytical results for the parallel planes [Eqs. (B35) and (B36)] and circular [Eq. 20 in [10]] geometries, one can see that the first-order $\mathcal{R}_{t}$ correction is $\mathrm{O}\left(\frac{k \mathcal{R}_{t}}{\gamma^{2}}\right)$, similar in magnitude to the $\mathcal{R}_{z}$ correction term [which is $\mathrm{O}\left(k \mathcal{R}_{z}\right)$ ].

Our algorithm calculates the impedance at small and at large frequency and in the perturbative region, for both nonultrarelativistic and ultrarelativistic regimes, as we show in the next section.

\section{B. Results}

We present results for a rectangular steel beam pipe with the conductivity $\sigma=0.23 \times 10^{7} \Omega^{-1} \mathrm{~m}^{-1}$ and dimension $2 a \times 2 b$. The longitudinal surface impedance $\mathcal{R}_{z}$ is given by Eq. (79) and $\mathcal{R}_{t}=-\mathcal{R}_{z}$. Ultrarelativistic, $\gamma=1000$, and nonultrarelativistic, $\gamma=1.42$, cases are considered. The vertical dimension is kept constant $b=3 \mathrm{~cm}$ while the horizontal one is varied such as the ratio $\frac{a}{b}$ increases from 1 to 3 . For $a=2 b$ we find that the impedance is already close to the corresponding parallel-plane limit.

First we benchmarked our algorithm by comparing the simulations for a parallel-plane beam pipe with the analytical results. The parallel-plane problem can be solved analytically as shown in Appendix B. We find that the algorithm converges to the exact results for $N$ of order of thousands.

In the ultrarelativistic regime and in the perturbative region defined outside the range of validity of Eqs. (80) and (81), the longitudinal impedance is proportional to $\omega^{\frac{1}{2}}$ while the transverse impedances are proportional to $\omega^{-\frac{1}{2}}$. The same behavior is known for circular and parallel-plane impedances. We define the proportionality coefficients $c_{l}$, $c_{w y}, c_{w x}, c_{s y}, c_{s x}$ by

$$
\begin{gathered}
Z^{\|}=c_{l} \frac{\mathcal{R}_{z}}{2 \pi b}, \\
\left(Z_{y}^{w}, Z_{x}^{w}, Z_{y}^{s}, Z_{x}^{s}\right)=\left(c_{w y}, c_{w x}, c_{s y}, c_{s x}\right) \frac{\mathcal{R}_{z}}{\pi k b^{3}},
\end{gathered}
$$

as in Yokoya's paper [18]. Our results agree with those presented by Yokoya [18], as can be seen in Fig. 1. Here we plot the coefficients on top of Fig. 8 from Ref. [18]. Note that the longitudinal impedance for a square

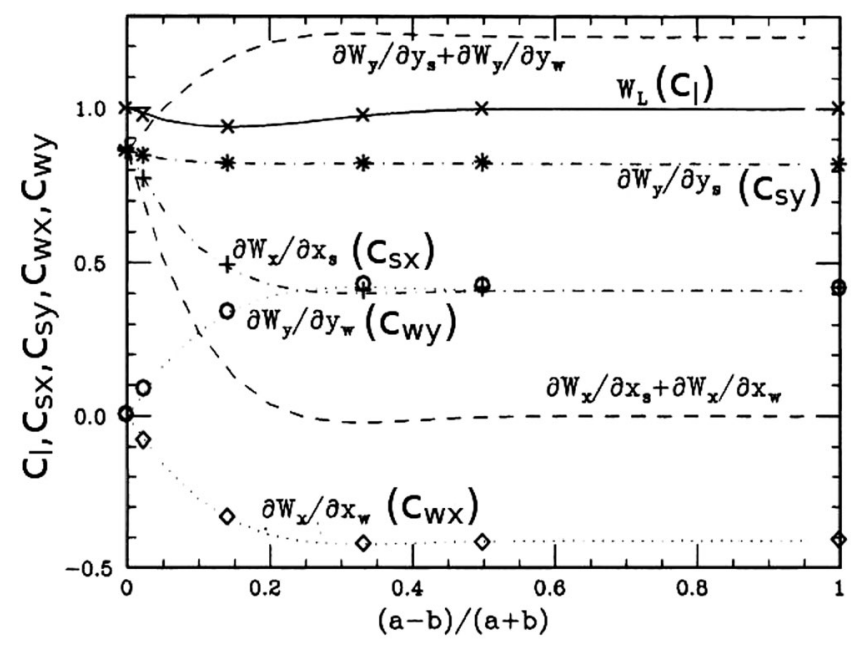

FIG. 1. Perturbative region defined outside the range of validity of Eqs. (80) and (81). Ultrarelativistic limit, $\gamma=$ 1000. The longitudinal impedance is proportional to $\omega^{\frac{1}{2}}$ while the transverse impedances are proportional to $\omega^{-\frac{1}{2}}$. The proportionality coefficients defined by Eqs. (82) and (83), $c_{l}$ (cross), $c_{s x}$ (plus), $c_{s y}$ (star), $c_{w x}$ (diamond), and $c_{w y}$ (circle), are plotted on top Yokoya's [18] Fig. 8. Note that the impedances for $\frac{a}{b}=2\left(\frac{a-b}{a+b}=0.33\right)$ are very close to the parallel-plane limit $\left(\frac{a-b}{a+b}=1\right)$. 


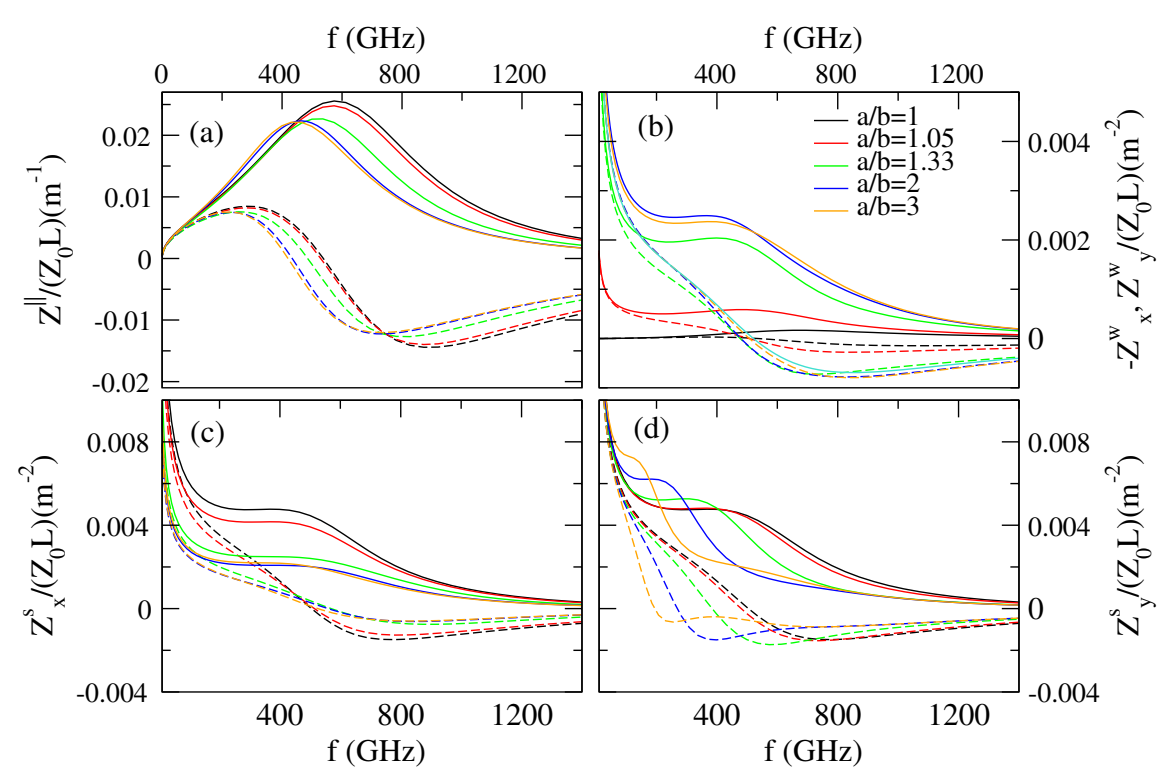

FIG. 2. Large frequency regime, $\gamma=1000$. The perturbation approximation fails when $f>\approx 60$ GHz. The real (imaginary) part of the impedance is plotted with solid (dashed) lines. At large frequencies the longitudinal impedance (a) decreases with increasing $\frac{a}{b}$, while the transverse impedances caused by the witness particle displacement (b) increase with increasing $\frac{a}{b}$. The transverse impedances caused by the source displacement (c) and (d) decrease with increasing $\frac{a}{b}$.

pipe, i.e., for $a=b$, is equal to that corresponding to a parallel-plane chamber, i.e., for $a \gg b$. Starting from a square pipe and increasing $\frac{a}{b}$, the longitudinal impedance decreases slightly until $\frac{a}{b} \approx 1.35\left(\frac{a-b}{a+b} \approx 0.15\right)$ where $c_{l}=$ 0.94 , and then increases asymptotically back. The transverse impedance caused by the witness particle displacement increases from zero to the value corresponding to the parallel-plane chamber when $\frac{a}{b}\left(\frac{a-b}{a+b}\right)$ is varied from 1 to 2 (0 to 0.33). Because of the Panofsky-Wenzel theorem, $Z_{x}^{w}=-Z_{y}^{w}$ in the ultrarelativistic limit. The zero value of $Z_{x}^{w}$ and $Z_{y}^{w}$ for a square pipe is a consequence of the large degree of symmetry [20]. The vertical transverse impedance caused by the source particle's displacement, $Z_{y}^{s}$, has a small dependence on $\frac{a}{b}$ while $Z_{x}^{s}$ decreases to half of its initial value when $\frac{a}{b}\left(\frac{a-b}{a+b}\right)$ is varied from 1 to 2 (0 to 0.33 ).

The ultrarelativistic impedance in the high frequency regime is presented in Fig. 2. For our parameters the perturbation theory fails when $f>\approx 60 \mathrm{GHz}$. At large frequencies the longitudinal impedance and the vertical impedances caused by the source displacement decrease with increasing $\frac{a}{b}$, while the transverse impedance caused by the witness particle displacement increases with increasing $\frac{a}{b}$.

Nonultrarelativistic effects dramatically change the impedance at high frequency. In Fig. 3 we show the impedance for $\gamma=1.42$. The $\omega^{\frac{1}{2}}$ behavior of the longitudinal impedance is valid up to only $\approx 0.4 \mathrm{GHz}$. Above this frequency the impedances corresponding to different values of $\frac{a}{b}$ are not proportional to each other. Above $1 \mathrm{GHz}$ the longitudinal impedance decreases with increasing $\frac{a}{b}$. The transverse impedances caused by the displacement of the witness particle increase with increasing $\frac{a}{b}$. Note that for the square beam pipe the transverse impedances $Z_{x}^{w}$ and $Z_{y}^{w}$ are nonzero, unlike in the ultrarelativistic case. Unlike the ultrarelativistic case, $Z_{x}^{w} \neq-Z_{y}^{w}$ as can be seen in Fig. 3(b). The horizontal transverse impedance caused by the source displacement, $Z_{x}^{s}$, Fig. 3(c), decreases with increasing $\frac{a}{b}$ while $Z_{y}^{s}$, Fig. 3(d), show negligible dependence of $\frac{a}{b}$.

The small-frequency nonperturbative regime for $\gamma=$ 1.42 is shown in Fig. 4. At small frequencies the nonultrarelativistic corrections are negligible aside a multiplicative factor of $\beta$ for the transverse impedance [14]. For our beam pipe parameters, this regime is effective for $f<\approx 100 \mathrm{KHz}$. An increase in the value of the wall surface impedance will, however, increase the characteristic frequency of this regime by a similar order of magnitude. The longitudinal impedance has a similar behavior to the one characteristic of the perturbative regime, i.e., a small decrease followed by an asymptotic increase when going from the square beam pipe to the parallel-planes limit. However, the longitudinal impedance has no perfect $\omega^{\frac{1}{2}}$ behavior and the difference between the real and the imaginary part is noticeable. The transverse impedances caused by the witness particle displacement, $-Z_{x}^{w}=Z_{y}^{w}$, increase from zero to the value corresponding to the parallel-planes limit. The horizontal impedances caused by the source displacement $Z_{x}^{s}$ decreases to half when going from the square beam pipe to parallel-plane limit. The vertical impedance $Z_{y}^{s}$ shows a small decrease 


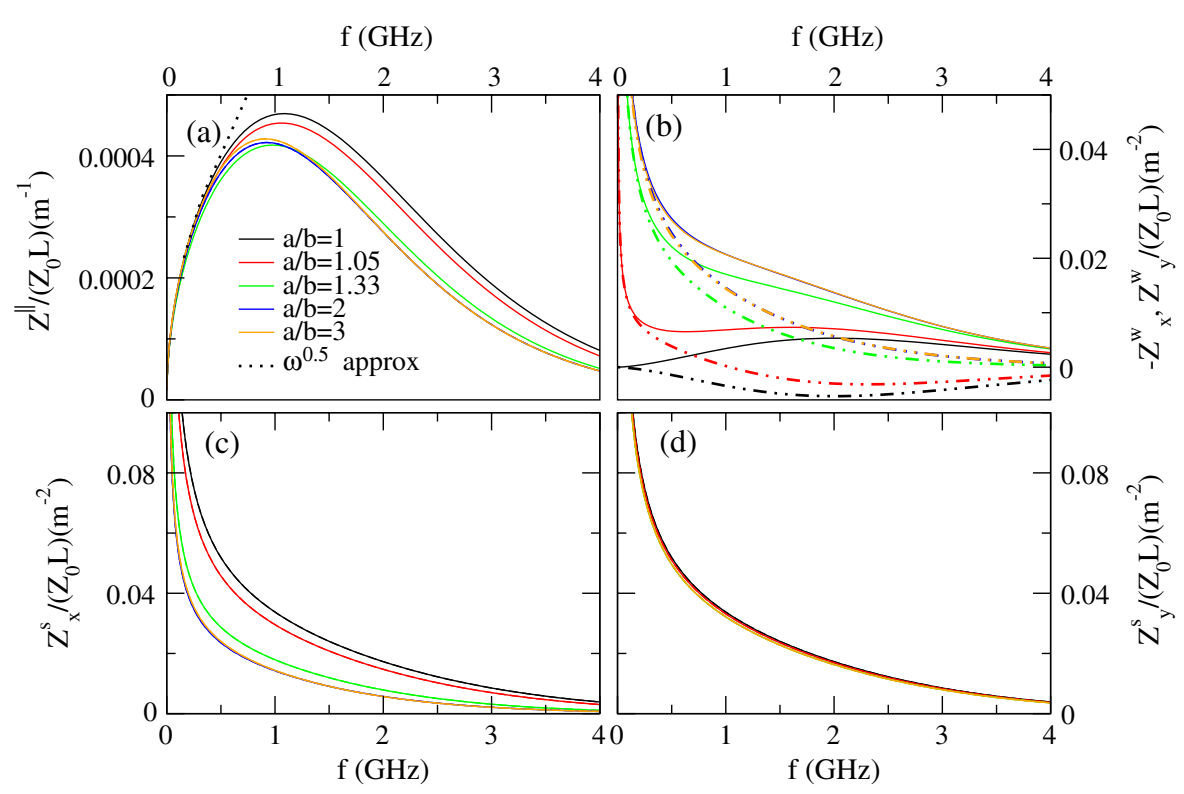

FIG. 3. Nonultrarelativistic impedance, $\gamma=1.42$. The real and the imaginary part of the impedance fall on top of each other. Finite $\gamma$ effects show for $f>\approx 0.4 \mathrm{GHz}$. The impedances corresponding to different values of $\frac{a}{b}$ are not proportional to each other. (a) Longitudinal impedance. At large frequencies (above $1 \mathrm{GHz}$ ) the longitudinal impedance decreases with increasing $\frac{a}{b}$. (b) The transverse impedances caused by the witness particle displacement, $-Z_{x}^{w}$ (dash-dotted line) and $Z_{y}^{w}$ (solid line), increase with increasing $\frac{a}{b}$. The plots for $\frac{a}{b}=2$ (blue) and $\frac{a}{b}=3$ (orange) are on top of each other. Note that $-Z_{x}^{w} \neq Z_{y}^{w}$, unlike the ultrarelativistic case. (c) The horizontal transverse impedance $Z_{x}^{s}$ decreases with increasing $\frac{a}{b}$. (d) The vertical transverse impedance $Z_{y}^{s}$ shows negligible dependence on $\frac{a}{b}$.

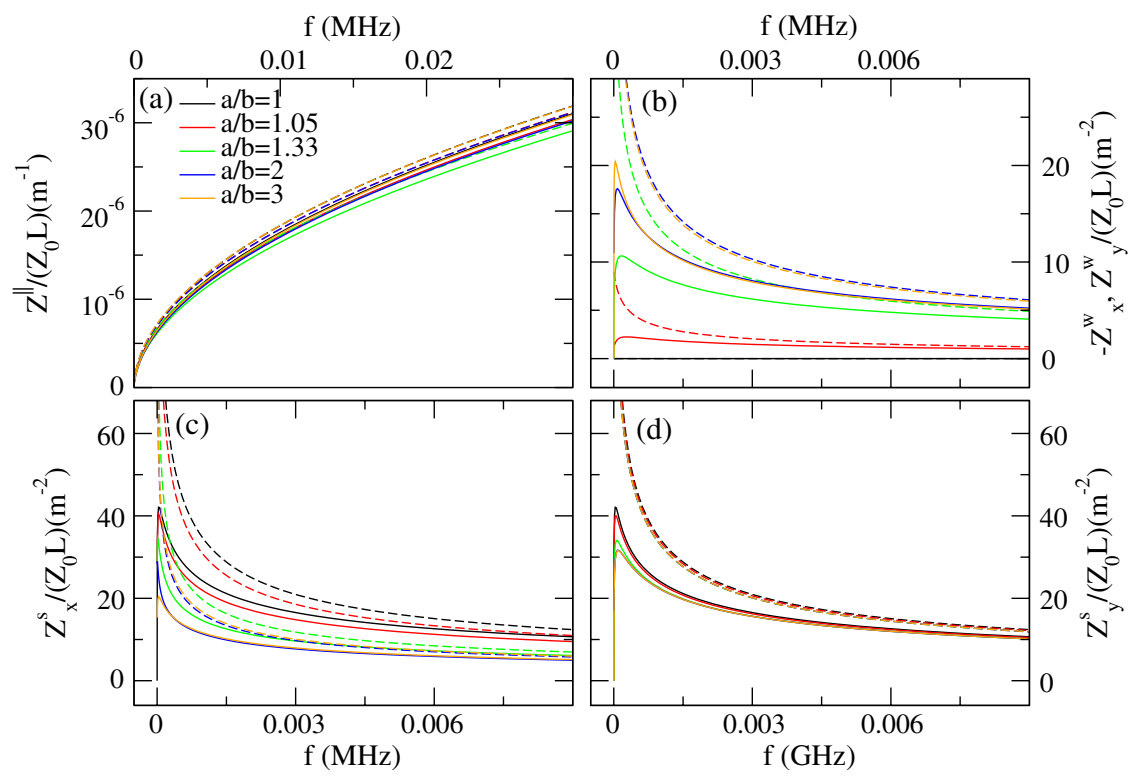

FIG. 4. Small-frequency nonperturbative region, $f<\approx 100 \mathrm{KHz} . \gamma=1.42$. The real part (solid line) and the imaginary part (dashed line) of impedances do not coincide. (a) Longitudinal impedance. (b) The transverse impedance caused by the witness particle displacement increases with increasing $\frac{a}{b}$. (c) The horizontal impedance $Z_{x}^{s}$ decreases with increasing $\frac{a}{b}$. The parallel plane $Z_{x}^{s}$ is about half of the square beam pipe $Z_{x}^{s}$. (d) The vertical impedance $Z_{y}^{s}$ shows a slight decrease with increasing $\frac{a}{b}$.

with increasing $\frac{a}{b}$. Note that our algorithm captures well the low frequency features of the transverse impedance, namely that the real part goes to zero and the imaginary part goes to a finite value when the frequency approaches zero [13].

\section{CONCLUSIONS}

We present an algorithm for calculating the impedance in beam pipes with arbitrary cross section. The method is nonperturbative, works at small and large frequencies, and 
does not assume the ultrarelativistic approximation. The equations for the electromagnetic potentials are discretized and the solution is obtained after solving a system of linear algebraic equations.

The impedance algorithm assumes that the electromagnetic field boundary conditions at the beam pipe wall are known and are provided as linear relations between the field transverse components. We describe an algorithm to calculate the boundary conditions for the general case of the multilayer beam pipe of arbitrary cross section.

Our simulations are checked against the analytical results for the parallel-plane beam pipe. We present an analytical derivation of the nonultrarelativistic parallelplane impedance as a function of wall surface impedance.

We show results for a rectangular metallic beam pipe, for both ultrarelativistic and finite- $\gamma$ cases. The ultrarelativistic perturbative regime is in perfect agreement with the work of Yokoya [18]. The rectangular longitudinal beam pipe impedance is proportional to $\omega^{\frac{1}{2}}$ while the rectangular transverse impedances behave as $\omega^{-\frac{1}{2}}$. This behavior is similar to the one characteristic of the circular and the parallel-plane beam pipes, the influence of the beam pipe geometry being captured by a renormalization factor. We find that this simple renormalization is not valid when the nonultrarelativistic effects are important or in the ultrarelativistic approximation at small and at large frequencies.

\section{ACKNOWLEDGMENTS}

This work was performed at Fermilab, operated by Fermi Research Alliance, LLC under Contract No. De-AC0207CH11359 with the United States Department of Energy. It was also supported by the ComPASS project, funded through the Scientific Discovery through Advanced Computing program in the DOE Office of High Energy Physics.

\section{APPENDIX A: ALGORITHM FOR THE WALL BOUNDARY CONDITIONS OF MULTILAYER STRUCTURES WITH ARBITRARY CROSS SECTION}

Our algorithm for the impedance calculation assumes knowledge of the wall boundary conditions in a form given by Eqs. (54) and (55). For an infinitely thick metallic beam pipe, the large wall conductivity limit makes it possible to use the boundary conditions in Eqs. (56) and (57). Nevertheless, in general one needs to calculate the boundary conditions by solving the electromagnetic problem outside the vacuum beam pipe. Here we present a numerical algorithm for calculating the wall boundary conditions for multilayer beam pipes with arbitrary cross section.

Let us assume that the medium outside the beam pipe is characterized by Ohm's law,

$$
\vec{j}=\sigma \vec{E}
$$

By choosing the gauge

$$
\nabla \vec{A}+\frac{\epsilon_{r} \mu_{r}}{c^{2}} \frac{\partial \Phi}{\partial t}+\mu \sigma \Phi=0
$$

the equations for the potentials can be written as

$$
\frac{\partial^{2} \Phi}{\partial x^{2}}+\frac{\partial^{2} \Phi}{\partial y^{2}}-\lambda^{2} \Phi=0
$$

and

$$
\frac{\partial^{2} \vec{A}}{\partial x^{2}}+\frac{\partial^{2} \vec{A}}{\partial y^{2}}-\lambda^{2} \vec{A}=0
$$

where

$$
\lambda^{2}=k^{2}\left(1-\epsilon_{r} \mu_{r} \beta^{2}\right)+i \omega \mu \sigma .
$$

The solution for the potentials can be expressed as before,

$$
\Phi(x, y)=\oint D_{\lambda}\left(x, y ; r_{l}\right) \Phi\left(r_{l}\right) d l-\oint G_{\lambda}\left(x, y ; r_{l}\right) \partial_{n} \Phi\left(r_{l}\right) d l,
$$

and analogously for $\vec{A}$. Fixing the gauge such that

$$
A_{z}=\epsilon_{r} \mu_{r} \frac{\beta}{c}\left(1-i \frac{\mu \sigma c^{2}}{\epsilon_{r} \mu_{r}}\right) \Phi,
$$

the same constraint as in the vacuum is implied for $A_{x}$ and $A_{y}$,

$$
\partial_{x} A_{x}+\partial_{y} A_{y}=0
$$

Following similar reasoning to that described in Sec. II A, one can consider two sets of independent variables $(\bar{\Phi}, \bar{A})$ to completely determine the solutions:

$$
\begin{gathered}
\bar{A}_{z}=g \bar{\Phi}, \\
\overline{\partial \Phi}=U \bar{\Phi}, \\
\overline{\partial A}_{x, y}=U \bar{A}_{x, y}, \\
\bar{A}_{x, y}=L_{x, y} \bar{A},
\end{gathered}
$$

where

$$
g=\epsilon_{r} \mu_{r} \frac{\beta}{c}\left(1-i \frac{\mu \sigma c^{2}}{\epsilon_{r} \mu_{r}}\right) .
$$

The matrices $U$ and $L_{x, y}$ are determined by the medium's properties and surface geometry.

Let us assume that the medium outside the beam pipe extends to infinity, i.e., that we have a one-layer problem. Only the surface potentials at the beam pipe wall are present in the field equations. Note that these surface potentials are different from the ones inside the beam pipe with only the tangential electromagnetic fields being continuous across the surface. The tangential fields at the surface read 


$$
\begin{gathered}
\bar{E}_{z}=i k(1-\beta c g) \bar{\Phi} \\
\bar{E}_{t}=-\partial_{\|} \bar{\Phi}-i \omega\left(t_{x} L_{x}+t_{y} L_{y}\right) \bar{A} \\
\mu_{r} Z_{0} \bar{H}_{z}=c\left[\left(t_{x} \partial_{\|}+n_{x} U\right) L_{y}-\left(t_{y} \partial_{\|}+n_{y} U\right) L_{x}\right] \bar{A} \\
\mu_{r} Z_{0} \bar{H}_{t}=\beta(\vec{t} \times \vec{n})_{z} U \bar{\Phi}+i k c\left(t_{x} L_{y}-t_{y} L_{x}\right) \bar{A}
\end{gathered}
$$

By inverting the set of equations given by Eqs. (A16) and (A17), one can write

$$
\left(\begin{array}{c}
\bar{\Phi} \\
\bar{A}
\end{array}\right)=\mathcal{H}\left(\begin{array}{l}
\bar{H}_{z} \\
\bar{H}_{t}
\end{array}\right)
$$

where $\mathcal{H}$ is a $2 N \times 2 N$ complex matrix. Using Eq. (A18), Eqs. (A14) and (A15) can be written as

$$
\left(\begin{array}{c}
\bar{E}_{z} \\
\bar{E}_{t}
\end{array}\right)=\mathcal{R}\left(\begin{array}{c}
\bar{H}_{z} \\
\bar{H}_{t}
\end{array}\right)
$$

Since the tangential fields are continuous across the surface, this equation represents the boundary condition used in Eqs. (54) and (55). Note that finding the boundary conditions for one-layer problem implies calculating the inverse of a $2 N \times 2 N$ complex matrix.

Next we consider a two-layer problem, where medium 1 outside the vacuum beam pipe has as its inner surface the vacuum beam pipe and as its outer surface medium 2 . We assume that medium 2 extends to infinity. We denote the fields and potentials inside medium 1 at the outer surface $\bar{E}_{z 1}, \bar{E}_{t 1}, \bar{H}_{z 1}, \bar{H}_{t 1}, \bar{\Phi}_{1}$, and $\bar{A}_{1}$, and at the inner surface $\bar{e}_{z 1}$, $\bar{e}_{t 1}, \bar{h}_{z 1}, \bar{h}_{t 1}, \bar{\phi}_{1}$, and $\bar{a}_{1}$. Equations (A10) and (A11) can be rewritten for the potentials at the outer surface as

$$
\begin{gathered}
\overline{\partial \Phi}_{1}=U_{1} \bar{\Phi}_{1}+u_{1} \bar{\phi}_{1}, \\
\overline{\partial A}_{x, y 1}=U_{1} \bar{A}_{x, y 1}+u_{1} \bar{a}_{x, y 1},
\end{gathered}
$$

where $U_{1}$ is a $N_{2} \times N_{2}$ matrix and $u_{1}$ is a $N_{2} \times N_{1}$ matrix. $N_{2}$ is the number of points at the outer surface while $N_{1}$ is the number of points at the inner surface.

The boundary condition at surface 2 (the outer surface of medium 1), is given by the equation

$$
\left(\begin{array}{c}
\bar{E}_{z 1} \\
\bar{E}_{t 1}
\end{array}\right)=\mathcal{R}_{2}\left(\begin{array}{c}
\bar{H}_{z 1} \\
\bar{H}_{t 1}
\end{array}\right) \text {, }
$$

where $\mathcal{R}_{2}$ can be calculated for medium 2 in the same way as $\mathcal{R}$ in Eq. (A19) was calculated in the previous one-layer example. The system of equations given by Eqs. (A20)-(A22) and the fixed gauge condition Eq. (A8) is similar to that given by Eqs. (36)-(38) and (22). One can regard the inner surface potentials $p_{1}=\left(\bar{\phi}_{1}, \bar{a}_{1}\right)$ as the source for the electromagnetic field inside medium 1 . Therefore, for the outer surface potentials $P_{1}=\left(\bar{\Phi}_{1}, \bar{A}_{1}\right)$ one gets an equation similar to Eq. (60):

$$
M_{1} P_{1}=S_{1} \text {, }
$$

where $M_{1}$ is a complex $2 N_{2} \times 2 N_{2}$ matrix containing information about the electromagnetic properties of medium 2. $S_{1}$ elements are proportional to $p_{1}$ just as $S$ is proportional to the source potential $\bar{\Phi}_{0}$ in Eq. (60).

By inverting $M_{1}$, one can write the solution as

$$
P_{1}=B p_{1} \text {, }
$$

where $B$ is a $2 N_{2} \times 2 N_{1}$ matrix. Using Eq. (A24) and the equation for the derivative of the potentials on the inner surface deduced from Eqs. (A10) and (A11),

$$
\begin{aligned}
& \overline{\partial \phi}_{1}=V_{1} \bar{\Phi}_{1}+v_{1} \bar{\phi}_{1}, \\
& \overline{\partial a}{ }_{1}=V_{1} \bar{A}_{1}+v_{1} \bar{a}_{1},
\end{aligned}
$$

one can write

$$
\partial p_{1}=u_{2} p_{1} \text {. }
$$

Since the outer potentials $\left(\bar{\Phi}_{1}, \bar{A}_{1}\right)$ have been eliminated, Eq. (A27) corresponds to Eqs. (A10) and (A11) for the one-layer problem. From this point one can proceed as in the one-layer problem.

Calculating the boundary conditions for a two-layer beam pipe requires inverting two $2 N_{2} \times 2 N_{2}$ and one $2 N_{1} \times 2 N_{1}$ complex matrices. In general any extra layer will add a new surface and will require inverting two extra $2 M \times 2 M$ complex matrices, where $M$ is the number of points necessary to describe the fields at the new surface.

\section{APPENDIX B: ANALYTICAL SOLUTION FOR NONULTRARELATIVISTIC PARALLEL-PLANE BEAM PIPE IMPEDANCE}

For a beam pipe with parallel-plane geometry, due to the translational symmetry along the horizontal direction, the different horizontal modes are decoupled and the problem can be solved analytically.

Consider a beam with a vertical offset $y=y_{0}$ moving along the $z$ direction between two parallel plates along the $x$ direction. The distance between the parallel plates is $2 b$.

The spectral decomposition along the horizontal direction can be written as

$$
\begin{aligned}
& \begin{aligned}
& {\left[\Phi(x, y), A_{y}(x, y), A_{z}(x, y)\right]=} \int_{-\infty}^{\infty}\left[\Phi(\eta, y), A_{y}(\eta, y), A_{z}(\eta, y)\right] \\
& \times \cos (\eta x) d \eta, \\
& A_{x}(x, y)=\int_{-\infty}^{\infty} A_{x}(\eta, y) \sin (\eta x) d \eta, \quad(\mathrm{B} 1)
\end{aligned} \\
& {\left[H_{x}(x, y), E_{y}(x, y), E_{z}(x, y)\right]} \\
& =\int_{-\infty}^{\infty}\left[H_{x}(\eta, y), E_{y}(\eta, y), E_{z}(\eta, y)\right] \cos (\eta x) d \eta, \\
& {\left[E_{x}(x, y), H_{y}(x, y), H_{z}(x, y)\right]} \\
& \quad=\int_{-\infty}^{\infty}\left[E_{x}(\eta, y), H_{y}(\eta, y), H_{z}(\eta, y)\right] \sin (\eta x) d \eta . \quad \text { (B2) }
\end{aligned}
$$


In Fourier space, $(\eta, y, k, \omega)$, Eqs. (3) and (4) read

$$
\begin{gathered}
\frac{\partial^{2} \Phi}{\partial y^{2}}-m^{2} \Phi=-\frac{\rho}{\epsilon_{0}} \delta\left(y-y_{0}\right), \\
\frac{\partial^{2} \vec{A}}{\partial y^{2}}-m^{2} \vec{A}=-\mu_{0} \beta c \rho \delta\left(y-y_{0}\right) \hat{z}
\end{gathered}
$$

where

$$
m^{2}=\eta^{2}+k^{2}-\frac{\omega^{2}}{c^{2}}=\eta^{2}+\frac{k^{2}}{\gamma^{2}} .
$$

The solution for the equation

$$
\left(\frac{\partial^{2}}{\partial y^{2}}-m^{2}\right) G(y)=\delta\left(y-y_{0}\right)
$$

is

$$
G(y)=-\frac{1}{2|m|} e^{-\left|m\left(y-y_{0}\right)\right|} .
$$

The potential equations are

$$
\begin{gathered}
\Phi=\frac{\rho}{2 \epsilon_{0}} \frac{e^{-m\left|\left(y-y_{0}\right)\right|}}{m}+a_{m} \cosh m y+\bar{a}_{m} \sinh m y, \\
A_{x}=m b_{m} \cosh m y+m \bar{b}_{m} \sinh m y, \\
A_{y}=-\eta \bar{b}_{m} \cosh m y-\eta b_{m} \sinh m y, \\
A_{z}=\frac{\beta}{c} \Phi,
\end{gathered}
$$

where we have imposed the gauge condition Eq. (22).

The electromagnetic field components are

$$
\begin{gathered}
E_{z}=i \frac{k}{\gamma^{2}}\left(\frac{\rho}{2 \epsilon_{0}} \frac{e^{-m\left|\left(y-y_{0}\right)\right|}}{m}+a_{m} \cosh m y+\bar{a}_{m} \sinh m y\right), \\
E_{x}=\frac{\eta}{m} \frac{\rho}{2 \epsilon_{0}} e^{-m\left|\left(y-y_{0}\right)\right|}+\left(\eta a_{m}-i k \beta c m b_{m}\right) \cosh m y \\
+\left(\eta \bar{a}_{m}-i k \beta c m \bar{b}_{m}\right) \sinh m y, \\
E_{y}=\operatorname{sgn}\left(y-y_{0}\right) \frac{\rho}{2 \epsilon_{0}} e^{-m\left|\left(y-y_{0}\right)\right|}-\left(m \bar{a}_{m}-i k \beta c \eta \bar{b}_{m}\right) \\
\quad \times \cosh m y-\left(m a_{m}-i k \beta c \eta b_{m}\right) \sinh m y, \\
Z_{0} H_{x}=-\operatorname{sgn}\left(y-y_{0}\right) \beta \frac{\rho}{2 \epsilon_{0}} e^{-m\left|\left(y-y_{0}\right)\right|} \\
\quad+\left(m \beta \bar{a}_{m}-i k c \eta \bar{b}_{m}\right) \cosh m y \\
\quad+\left(m \beta a_{m}-i k c \eta b_{m}\right) \sinh m y,
\end{gathered}
$$

$$
\begin{gathered}
Z_{0} H_{y}=\frac{\eta \beta}{m} \frac{\rho}{2 \epsilon_{0}} e^{-m\left|\left(y-y_{0}\right)\right|}+\left(\eta \beta a_{m}-i k c m b_{m}\right) \cosh m y \\
+\left(\eta \beta \bar{a}_{m}-i k c m \bar{b}_{m}\right) \sinh m y, \\
Z_{0} H_{z}=-c \frac{k^{2}}{\gamma^{2}}\left(\bar{b}_{m} \cosh m y+b_{m} \sinh m y\right) .
\end{gathered}
$$

The coefficients $a_{m}, \bar{a}_{m}, b_{m}$, and $\bar{b}_{m}$ are to be determined from the boundary conditions at the chamber walls. The boundary conditions are given by the surface wall impedance,

$$
\mathcal{R}_{z}(\eta)= \pm\left.\frac{E_{z}(\eta)}{H_{x}(\eta)}\right|_{y= \pm b}
$$

$$
R_{x}(\eta)=\left.\mp \frac{E_{x}(\eta)}{H_{z}(\eta)}\right|_{y= \pm b}
$$

which imply

$$
\begin{gathered}
E_{z}(b)+E_{z}(-b)=\mathcal{R}_{z}\left[H_{x}(b)-H_{x}(-b)\right], \\
E_{z}(b)-E_{z}(-b)=\mathcal{R}_{z}\left[H_{x}(b)+H_{x}(-b)\right], \\
E_{x}(b)+E_{x}(-b)=-\mathcal{R}_{x}\left[H_{z}(b)-H_{z}(-b)\right], \\
E_{x}(b)-E_{x}(-b)=-\mathcal{R}_{x}\left[H_{z}(b)+H_{z}(-b)\right] .
\end{gathered}
$$

These equations yield two systems of two independent linear equations. From Eqs. (B20) and (B22), one has

$$
\begin{gathered}
a_{m}\left(-i \frac{k}{\gamma^{2}} \cosh m b+\frac{\mathcal{R}_{z}}{Z_{0}} \beta m \sinh m b\right) \\
-b_{m} i k c \eta \frac{\mathcal{R}_{z}}{Z_{0}} \sinh m b \\
=\frac{\rho \cosh m y_{0}}{2 \epsilon_{0}} e^{-m b}\left(i \frac{k}{m \gamma^{2}}+\beta \frac{\mathcal{R} z}{Z_{0}}\right), \\
a_{m} \eta \cosh m b-b_{m}\left(i k \beta c m \cosh m b+\frac{\mathcal{R}_{x}}{Z_{0}} c \frac{k^{2}}{\gamma^{2}} \sinh m b\right) \\
=-\frac{\rho \cosh m y_{0}}{2 \epsilon_{0}} e^{-m b} \frac{\eta}{m},
\end{gathered}
$$

with solution 


$$
\xi_{\eta}=i \frac{k}{\gamma^{2}} a_{m}=-\frac{\rho \cosh m y_{0}}{2 \epsilon_{0}} \frac{\frac{\eta^{2}}{m^{2}} \frac{\mathcal{R}_{z}}{\beta Z_{0}} \operatorname{sech}^{2} m b+\left\{\frac{1}{\gamma^{2}}\left[i \frac{k}{m}+\left(\frac{k^{2}}{m^{2}}-1\right) \frac{\mathcal{R}_{z}}{\beta Z_{0}}-i \frac{k}{m} \frac{\mathcal{R}_{z} \mathcal{R}_{x}}{Z_{0}^{2}} \tanh m b\right]+\frac{1}{\gamma^{4}} \frac{k^{2}}{m^{2}} \frac{\mathcal{R}_{x}}{\beta Z_{0}} \tanh m b\right\} e^{-m b} \operatorname{sech} m b}{1+i \frac{\mathcal{R}_{z}}{\beta Z_{0}}\left(\frac{k}{m}-\frac{m}{k}\right) \tanh m b+\frac{\mathcal{R}_{z} \mathcal{R}_{x}}{Z_{0}^{2}} \tanh ^{2} m b-\frac{i}{\gamma^{2}} \frac{\mathcal{R}_{x}}{\beta Z_{0}} \frac{k}{m} \tanh m b}
$$

Equations (B21) and (B23) yield

$$
\begin{gathered}
\bar{a}_{m}\left(-i \frac{k}{\gamma^{2}} \sinh m b+\frac{\mathcal{R}_{z}}{Z_{0}} \beta m \cosh m b\right)-\bar{b}_{m} i k c \eta \frac{\mathcal{R}_{z}}{Z_{0}} \cosh m b=\frac{\rho \sinh m y_{0}}{2 \epsilon_{0}} e^{-m b}\left(i \frac{k}{m \gamma^{2}}+\beta \frac{\mathcal{R}_{z}}{Z_{0}}\right) \\
\bar{a}_{m} \eta \sinh m b-b_{m}\left(i k \beta c m \sinh m b+\frac{\mathcal{R}_{x}}{Z_{0}} c \frac{k^{2}}{\gamma^{2}} \cosh m b\right)=-\frac{\rho \sinh m y_{0}}{2 \epsilon_{0}} e^{-m b} \frac{\eta}{m}
\end{gathered}
$$

with solution

$$
\bar{\xi}_{\eta}=i \frac{k}{\gamma^{2}} \bar{a}_{m}=-\frac{\rho \sinh m y_{0}}{2 \epsilon_{0}} \frac{\frac{\eta^{2}}{m^{2}} \frac{\mathcal{R}_{z}}{\beta Z_{0}} \operatorname{csch}^{2} m b+\left\{\frac{1}{\gamma^{2}}\left[i \frac{k}{m}+\left(\frac{k^{2}}{m^{2}}-1\right) \frac{\mathcal{R}_{z}}{\beta Z_{0}}-i \frac{k}{m} \frac{\mathcal{R}_{z} \mathcal{R}_{x}}{Z_{0}^{2}} \operatorname{coth} m b\right]+\frac{1}{\gamma^{4}} \frac{k^{2}}{m^{2}} \frac{\mathcal{R}_{x}}{\beta Z_{0}} \operatorname{coth} m b\right\} e^{-m b} \operatorname{csch} m b}{1+i \frac{\mathcal{R}_{z}}{\beta Z_{0}}\left(\frac{k}{m}-\frac{m}{k}\right) \operatorname{coth} m b+\frac{\mathcal{R}_{z} \mathcal{R}_{x}}{Z_{0}^{2}} \operatorname{coth}^{2} m b-\frac{i}{\gamma^{2}} \frac{\mathcal{R}_{x}}{\beta Z_{0}} \frac{k}{m} \operatorname{coth} m b} .
$$

Note that the longitudinal component of the electric field is

$$
E_{z}=\xi_{\eta} \cosh m y+\bar{\xi}_{\eta} \sinh m y+\frac{i}{\gamma^{2}} \frac{\rho}{2 \epsilon_{0}} e^{-\left|m\left(y-y_{0}\right)\right|} \frac{k}{|m|} .
$$

For an ideal conducting beam pipe $\left(\mathcal{R}_{z}=\mathcal{R}_{x}=0\right)$, the electric field is

$$
E_{z}^{\infty}=\xi_{\eta}^{\infty} \cosh m y+\bar{\xi}_{\eta}^{\infty} \sinh m y+\frac{i}{\gamma^{2}} \frac{\rho}{2 \epsilon_{0}} e^{-\left|m\left(y-y_{0}\right)\right|} \frac{k}{|m|}
$$

where

$$
\begin{aligned}
& \xi_{\eta}^{\infty}=-i \frac{k}{\gamma^{2} m} \frac{\rho \cosh m y_{0}}{2 \epsilon_{0}} e^{-m b} \operatorname{sech} m b, \\
& \bar{\xi}_{\eta}^{\infty}=-i \frac{k}{\gamma^{2} m} \frac{\rho \sinh m y_{0}}{2 \epsilon_{0}} e^{-m b} \operatorname{csch} m b
\end{aligned}
$$

The finite-conductivity contribution to the electric field is

$$
E_{z}^{\sigma}=E_{z}-E_{z}^{\infty}=\xi_{\eta}^{\sigma} \cosh m y+\bar{\xi}_{\eta}^{\sigma} \sinh m y,
$$

where

$$
\xi_{\eta}^{\sigma}=\xi_{\eta}-\xi_{\eta}^{\infty}=-\frac{\rho \cosh m y_{0}}{2 \epsilon_{0}} \operatorname{sech}^{2} m b \frac{\frac{\mathcal{R}_{z}}{\beta Z_{0}} \frac{\eta^{2}}{m^{2}}+\frac{1}{\gamma^{2}}\left[\frac{\mathcal{R}_{z}}{\beta Z_{0}}\left(\frac{k^{2}}{m^{2}}-1\right)-i \frac{\mathcal{R}_{z} \mathcal{R}_{x}}{Z_{0}^{2}} \frac{k}{m} \tanh m b\right]}{1+i \frac{\mathcal{R}_{z}}{\beta Z_{0}}\left(\frac{k}{m}-\frac{m}{k}\right) \tanh m b+\frac{\mathcal{R}_{z} \mathcal{R}_{x}}{Z_{0}^{2}} \tanh ^{2} m b-\frac{i}{\gamma^{2}} \frac{\mathcal{R}_{x}}{\beta Z_{0}} \frac{k}{m} \tanh m b},
$$

and

$$
\bar{\xi}_{\eta}^{\sigma}=\bar{\xi}_{\eta}-\bar{\xi}_{\eta}^{\infty}=-\frac{\rho \sinh m y_{0}}{2 \epsilon_{0}} \operatorname{csch}^{2} m b \frac{\frac{\mathcal{R}_{z}}{\beta Z_{0}} \frac{\eta^{2}}{m^{2}}+\frac{1}{\gamma^{2}}\left[\frac{\mathcal{R}_{z}}{\beta Z_{0}}\left(\frac{k^{2}}{m^{2}}-1\right)-i \frac{\mathcal{R}_{z} \mathcal{R}_{x}}{Z_{0}^{2}} \frac{k}{m} \operatorname{coth} m b\right]}{1+i \frac{\mathcal{R}_{z}}{\beta Z_{0}}\left(\frac{k}{m}-\frac{m}{k}\right) \operatorname{coth} m b+\frac{\mathcal{R}_{z} \mathcal{R}_{x}}{Z_{0}^{2}} \operatorname{coth}^{2} m b-\frac{i}{\gamma^{2}} \frac{\mathcal{R}_{x}}{\beta Z_{0}} \frac{k}{m} \operatorname{coth} m b} .
$$

\section{Longitudinal impedance}

The resistive wall longitudinal impedance is

$$
Z^{\|}(\omega)=-\frac{E_{z}^{\sigma}\left(x=y=y_{0}=0\right)}{\rho \beta c}=-\frac{1}{\pi \rho \beta c} \int_{0}^{\infty} d \eta \xi_{\eta}^{\sigma}
$$




\section{Horizontal transverse impedance}

The derivative of the horizontal Lorentz force, $F_{x}$, with respect to the witness particle's displacement is

$$
\frac{1}{q} \frac{\partial F_{x}}{\partial x}=-\frac{1}{\gamma^{2}} \partial_{x}^{2} \Phi=i \frac{\eta^{2}}{k} E_{z} .
$$

The horizontal impedance is

$$
\begin{aligned}
Z_{x}^{s} & =-Z_{x}^{w}=\frac{1}{i q \rho \beta c} \frac{\partial F_{x}}{\partial x}\left(x=y=y_{0}=0\right) \\
& =-\frac{1}{\pi \rho \beta c k} \int_{0}^{\infty} d \eta \eta^{2} \xi_{\eta} .
\end{aligned}
$$

Finally, the ideal conducting beam pipe impedance is

$$
\begin{aligned}
Z_{x}^{s \infty} & =-\frac{1}{\pi \rho \beta c k} \int_{0}^{\infty} d \eta \eta^{2} \xi_{\eta}^{\infty} \\
& =\frac{j}{\gamma^{2}} \frac{Z_{0}}{2 \pi \beta} \int_{0}^{\infty} d \eta \frac{\eta^{2}}{m} e^{-m b} \operatorname{sech} m b .
\end{aligned}
$$

Note that for large $\gamma$, when $m \approx \eta$,

$$
\begin{aligned}
Z_{x}^{s \infty} & =-Z_{x}^{w \infty}=\frac{i}{\gamma^{2}} \frac{Z_{0}}{2 \pi \beta} \int_{0}^{\infty} d \eta \eta e^{-\eta b} \operatorname{sech} \eta b \\
& =\frac{i}{\gamma^{2}} \frac{Z_{0}}{2 \pi \beta b^{2}} \frac{\pi^{2}}{24},
\end{aligned}
$$

in agreement with Laslett's calculations [24], while the resistive wall horizontal impedance is

$$
Z_{x}^{s \sigma}=-Z_{x}^{w \sigma}=-\frac{1}{\pi \rho \beta c k} \int_{0}^{\infty} d \eta \eta^{2} \xi_{\eta}^{\sigma} .
$$

\section{Vertical impedance}

The vertical Lorentz force is

$$
\begin{aligned}
\frac{F_{y}}{q}= & -\frac{1}{\gamma^{2}} \partial_{y} \Phi=\frac{i}{k} \partial_{y} E_{z} \\
= & \frac{i m}{k}\left(\xi_{\eta} \sinh m y+\bar{\xi}_{\eta} \cosh m y\right) \\
& +\operatorname{sgn}\left(y-y_{0}\right) \frac{1}{\gamma^{2}} \frac{\rho}{2 \epsilon_{0}} e^{-\left|m\left(y-y_{0}\right)\right|}
\end{aligned}
$$

The source transverse impedance is

$$
\begin{aligned}
Z_{y}^{s} & =-\frac{1}{i q \rho \beta c} \frac{\partial F_{y}}{\partial y_{0}}\left(x=y=0=y_{0}=0\right) \\
& =-\frac{1}{\pi \rho \beta c k} \int_{0}^{\infty} d \eta m \frac{\partial \bar{\xi}}{\partial y_{0}}\left(y_{0}=0\right) .
\end{aligned}
$$

Finally, the ideally conducting beam pipe impedance is

$$
\begin{aligned}
Z_{y}^{s \infty} & =-\frac{1}{\pi \rho \beta c k} \int_{0}^{\infty} d \eta m \frac{\partial \bar{\xi}^{\infty}}{\partial y_{0}}\left(y_{0}=0\right) \\
& =\frac{i}{\gamma^{2}} \frac{Z_{0}}{2 \pi \beta} \int_{0}^{\infty} d \eta m e^{-m b} \operatorname{csch} m b=\frac{i}{\gamma^{2}} \frac{Z_{0}}{2 \pi \beta b^{2}} \frac{\pi^{2}}{12}
\end{aligned}
$$

again in agreement with Laslett's calculations [24].

The witness transverse impedance is

$$
\begin{aligned}
Z_{y}^{w} & =-\frac{1}{i q \rho \beta c} \frac{\partial F_{y}}{\partial y}\left(x=y=0=y_{0}=0\right) \\
& =-\frac{1}{\pi \rho \beta c k} \int_{0}^{\infty} d \eta m^{2} \xi_{\eta} .
\end{aligned}
$$

Note that in the ultrarelativistic limit, i.e., when $m=\eta$, $Z_{y}^{w}=-Z_{x}^{w}$, in agreement with the Panofsky-Wenzel theorem.

\section{APPENDIX C: $6 N$ INDEPENDENT VARIABLES ALGORITHM}

Although it is not necessarily the most efficient way to calculate the potentials, we present here a straightforward approach which considers all $6 N$ variables, $\bar{\Phi}_{i}, \bar{A}_{x i}, \bar{A}_{y i}$, $\bar{\partial}_{i}, \overline{\partial A}_{x i}, \overline{\partial A}_{y i}, i=\overline{1, N}$, to be independent. This approach offers a broader view of the equations' structure and might turn to be useful for numerical optimization and parallelization for problems where large $N$ is required for convergence.

The fixed gauge condition, Eq. (22), on the surface reads

$$
t_{x} \partial_{\|} \bar{A}_{x}+n_{x} \overline{\partial A}_{x}+t_{y} \partial_{\|} \bar{A}_{y}+n_{y} \overline{\partial A}_{y}=0 .
$$

The electromagnetic fields at the wall reads

$$
\bar{E}_{z}=i \frac{k}{\gamma^{2}} \bar{\Phi}
$$

$$
\begin{gathered}
\bar{E}_{x}=-t_{x} \partial_{\|} \bar{\Phi}-n_{x} \overline{\partial \Phi}-i \omega \bar{A}_{x}, \\
\bar{E}_{y}=-t_{y} \partial_{\|} \bar{\Phi}-n_{y} \overline{\partial \Phi}-i \omega \bar{A}_{y}, \\
Z_{0} \bar{H}_{x}=\beta t_{y} \partial_{\|} \bar{\Phi}+\beta n_{y} \bar{\partial} \bar{\Phi}-i c k \bar{A}_{y}, \\
Z_{0} \bar{H}_{y}=-\beta t_{x} \partial_{\|} \bar{\Phi}-\beta n_{x} \overline{\partial \Phi}+i c k \bar{A}_{x},
\end{gathered}
$$

$$
Z_{0} \bar{H}_{z}=c\left(t_{x} \partial_{\|} \bar{A}_{y}+n_{x} \overline{\partial A}_{y}-t_{y} \partial_{\|} \bar{A}_{x}-n_{y} \overline{\partial A}_{x}\right) .
$$

The boundary conditions [Eqs. (56) and (57)] imply

$i \frac{k}{\gamma^{2}} \bar{\Phi}-\frac{\mathcal{R}_{z}}{Z_{0}} \beta\left(t_{x} n_{y}-t_{y} n_{x}\right) \overline{\partial \Phi}-i k c \frac{\mathcal{R}_{z}}{Z_{0}}\left(t_{x} \bar{A}_{y}-t_{y} \bar{A}_{x}\right)=0$, 


$$
\begin{aligned}
& -\partial_{\|} \bar{\Phi}-\left(j \omega t_{x}-\frac{\mathcal{R}_{t}}{Z_{0}} c t_{y} \partial_{\|}\right) \bar{A}_{x}-\left(j \omega t_{y}+\frac{\mathcal{R}_{t}}{Z_{0}} c t_{x} \partial_{\|}\right) \bar{A}_{y} \\
& +\frac{\mathcal{R}_{t}}{Z_{0}} c\left(n_{y} \overline{\partial A}_{x}-n_{x} \overline{\partial A}_{y}\right)=0
\end{aligned}
$$

The equations (C1), (C8), and (C9), together with Eqs. (30)-(32) yield

$$
M P=S
$$

with

$$
\left(\begin{array}{cccccc}
M_{00} & M_{01} & 0 & 0 & 0 & 0 \\
0 & 0 & M_{12} & M_{13} & 0 & 0 \\
0 & 0 & 0 & 0 & M_{24} & M_{25} \\
0 & 0 & M_{32} & M_{33} & M_{34} & M_{35} \\
M_{40} & 0 & M_{42} & M_{43} & M_{44} & M_{45} \\
M_{50} & M_{51} & M_{52} & 0 & M_{54} & 0
\end{array}\right)\left(\begin{array}{c}
\bar{\Phi} \\
\overline{\partial \Phi} \\
\bar{A}_{x} \\
\overline{\partial A}_{x} \\
\bar{A}_{y} \\
\overline{\partial A}_{y}
\end{array}\right)=\left(\begin{array}{c}
-\bar{\Phi}_{0} \\
0 \\
0 \\
0 \\
0 \\
0
\end{array}\right)
$$

where $P=\left(\bar{\Phi}, \overline{\partial \Phi}, \bar{A}_{x}, \overline{\partial A}_{x}, \bar{A}_{y}, \overline{\partial A}_{y}\right)$ and $S=\left(-\bar{\Phi}_{0}, 0,0\right.$, $0,0)$ are vectors of length $6 N . M_{m n}$ represents a $N \times N$ block matrix, with the specific values

$$
\begin{gathered}
M_{00}=M_{12}=M_{34}=D-I, \\
M_{01}=M_{13}=M_{35}=-G, \\
M_{32 i j}=t_{x i} \partial_{\|}(i, j), \\
M_{33 i j}=n_{x i} \delta(i, j), \\
M_{34 i j}=t_{y i} \partial_{\|}(i, j), \\
M_{35 i j}=n_{y i} \delta(i, j), \\
M_{40 i j}=-\partial_{\|}(i, j), \\
M_{42 i j}=-j \omega t_{x i} \delta(i, j)+\frac{\mathcal{R}_{t i}}{Z_{0}} c t_{y i} \partial_{\|}(i, j), \\
M_{43 i j}=\frac{\mathcal{R}_{t i}}{Z_{0}} c n_{y i} \delta(i, j), \\
M_{50 i j}=i \frac{\mathcal{R}_{z i}}{Z_{0}^{2}} \delta(i, j), \\
M_{45 i j}=-\frac{\mathcal{R}_{t i}}{Z_{0}} c n_{x i} \delta(i, j), \\
Z_{0} c t_{x i} \partial_{\|}(i, j),
\end{gathered}
$$

$$
\begin{gathered}
M_{52 i j}=i k c \frac{\mathcal{R}_{z i}}{Z_{0}} t_{y i} \delta(i, j), \\
M_{54 i j}=-i k c \frac{\mathcal{R}_{z i}}{Z_{0}} t_{x i} \delta(i, j) .
\end{gathered}
$$

[1] S. A. Heifets and S. A. Kheifets, Rev. Mod. Phys. 63, 631 (1991).

[2] B. Zotter and S. A. Kheifets, Impedances and Wakes in High-Energy Particle Accelerators (World Scientific Publishing, Singapore, 1998).

[3] R. Gluckstern, CERN Report No. 2000-011, 2000.

[4] A. Chao, Physics of Collective Beam Instabilities in High Energy Accelerators (John Wiley \& Sons, Inc., New York, 1993).

[5] A. M. Al-Khateeb, O. Boine-Frankenheim, I. Hofmann, and Giovanni Rumolo, Phys. Rev. E 63, 026503 (2001).

[6] F. Zimmermann and K. Oide, Phys. Rev. ST Accel. Beams 7, 044201 (2004).

[7] A. M. Al-Khateeb, O. Boine-Frankenheim, R. W. Hasse, and I. Hofmann, Phys. Rev. E 71, 026501 (2005).

[8] A. M. Al-Khateeb, R. W. Hasse, O. Boine-Frankenheim, W. M. Daqa, and I. Hofmann, Phys. Rev. ST Accel. Beams 10, 064401 (2007).

[9] N. Wang and Q. Qin, Phys. Rev. ST Accel. Beams 10, 111003 (2007).

[10] H. Hahn, Phys. Rev. ST Accel. Beams 13, 012002 (2010).

[11] N. Mounet and E. Metral, Report No. CERN-BE-2009039, 2009.

[12] A. Burov and V. Lebedev, in Proceedings of the 8th European Particle Accelerator Conference, Paris, 2002 (EPS-IGA and CERN, Geneva, 2002), p. 1452.

[13] K. Y. Ng, Fermilab Report No. FN-0744, 2004.

[14] A. Burov and V. Lebedev, Fermilab Report No. FERMILAB-CONF-02-101-T, 2002.

[15] N. Mounet and E. Metral, Report No. CERN-ATS-Note2010-056 TECH, 2010.

[16] A. Macridin, P. Spentzouris, J. Amundson, L. Spentzouris, and D. McCarron, Phys. Rev. ST Accel. Beams 14, 061003 (2011).

[17] R. Gluckstern, J. van Zeijts, and B. Zotter, Phys. Rev. E 47, 656 (1993).

[18] Kaoru Yokoya, Part. Accel. 41, 221 (1993).

[19] S. Kurennoy, EPAC Conf. Proc. 96 C960610, 1449-1451 (1996), TUP118G.

[20] S. Heifets, A. Wagner, B. Zotter, Report No. SLAC/ AP110, 1998.

[21] J. D. Jackson, Classical Electrodynamics (John Wiley \& Sons, Inc., New York, 1999), 3rd ed.

[22] A. Chao, S. Heifets, and Bruno Zotter, Phys. Rev. ST Accel. Beams 5, 111001 (2002).

[23] K. Bane, Report No. SLAC-AP-87, 1991; K. Bane and M. Sands, in Proceedings of the Micro Bunches Workshop (1995).

[24] L. J. Laslett, Report No. RNL-7534, p. 325. 